\title{
P334: Catalysing local production of alcohol based handrub in african hospitals: the power of south-north-south partnership
}

\author{
L Bengaly ${ }^{1 *}$, JD Hightower ${ }^{2}$, P Bonnabry ${ }^{3}$, SB Syed ${ }^{4}$ \\ From 2nd International Conference on Prevention and Infection Control (ICPIC 2013) \\ Geneva, Switzerland. 25-28 June 2013
}

\begin{abstract}
Introduction
The $58^{\text {th }}$ WHO African Regional Committee in 2008 called for action in 12 patient safety action areas, including infection prevention and control (IPC). Hand hygiene through use of alcohol based handrub (ABHR) is a clear mechanism to translate a regional mandate into local action. However, ABHR availability is a key challenge in African hospitals.
\end{abstract}

\section{Objectives}

To describe how south-north-south partnership mechanisms can be utilized to enhance capacity in the local production of ABHR in African hospitals.

\section{Methods}

On-going activity reports from the WHO African Partnerships for Patient Safety (APPS) were examined to capture key transferable lessons for the global IPC community. The focus of the examination was twofold: mechanism of partnership functioning and potential for replication.

\section{Results}

Four key steps were identified in partnership functioning. First, develop expertise in a hospital in Mali through a north-south partnership with Geneva University Hospitals. Second, develop capacity in APPS partnership hospitals in Senegal, Mali, Cameroon through a south-north-south mechanism. Third, test ABHR production workshop design through capacity-building in multiple hospitals in Yaoundé, Cameroon through a Mali-Cameroon partnership. Fourth, scale-up capacity in 6 further African countries (Ghana, Mozambique, Rwanda, Tanzania, Zambia,
Zimbabwe) through a multi-country south-south ABHR production workshop led by Malian expertise and involving APPS hospitals in each country. The end product from this multi-step process is a practical ABHR production workshop with quality control in-built, designed through partnership and available for wide replication in the African Region.

\section{Conclusion}

Calls for patient safety and IPC improvement can be considered hollow in the face of structural challenges in many African hospitals. One emerging solution is the development of country-based capacity in ABHR production through the use of a south-north-south partnership mechanism. The co-designed practical ABHR production workshop can now be utilized for local in-country action on hand hygiene through ABHR production.

\section{Disclosure of interest}

None declared.

\section{Author details}

${ }^{1}$ Pharmacy, CHU Hospital Gabriel Touré, Bamako, Mali. ${ }^{2}$ African Partnerships for Patient Safety, World Health Organization, Harare, Zimbabwe. ${ }^{3}$ Pharmacie des HUG, Geneva University Hospitals, Geneva, Switzerland. "WHO Patient Safety, World Health Organization, Geneva, Switzerland.

Published: 20 June 2013

doi:10.1186/2047-2994-2-S1-P334

Cite this article as: Bengaly et al:: P334: Catalysing local production of alcohol based handrub in african hospitals: the power

of south-north-south partnership. Antimicrobial Resistance and Infection Control 2013 2(Suppl 1):P334.

'Pharmacy, CHU Hospital Gabriel Touré, Bamako, Mali

Full list of author information is available at the end of the article

(c) 2013 Bengaly et al; licensee BioMed Central Ltd. This is an Open Access article distributed under the terms of the Creative Commons Attribution License (http://creativecommons.org/licenses/by/2.0), which permits unrestricted use, distribution, and reproduction in any medium, provided the original work is properly cited. 